$\triangle$ CTA $\mathbb{N} E O P H I L O L O G I C A$

DOI: 10.4312/an.48.1-2.87-99

UDK: 82.0-2:821.112.2.09Brecht B.

\title{
Zur Rolle der Emotionen in Brechts Dramentheorie
}

\section{Špela Virant}

\begin{abstract}
Mitte des 19. Jahrhunderts begann ein Prozess der 'Abkühlung' in den Künsten. Die dargestellten Gefühle und die gefühlvolle Darstellungsweise wurden aus der Literatur verdrängt oder sehr nüchtern betrachtet. Es kommt zu einer Rationalisierung, Ökonomisierung und Ideologisierung der gesellschaftlichen Emotionsdiskurse, was auch die Dramentheorie beeinflusst, für die die Erregung und Reinigung der Gefühle seit der Poetik von Aristoteles von zentraler Bedeutung ist. Brecht bekämpfte das aristotelische Theater aus ideologischen und politischen Gründen. Je mehr er gegen Aristoteles argumentierte, desto stärker näherte er sich einer modernen Lesart der Poetik an. Interessant ist aber, dass sich auch das sogenannte postdramatische Theater, das sich von Aristoteles und Brecht ausdrücklich distanziert, immer noch als kalt bezeichnet.
\end{abstract}

Key words: Bertolt Brecht, Aristoteles, Poetik, Dramentheorie, Theatertheorie 
In der Dramentheorie spielen, seit Aristoteles in seiner Poetik die Katharsis als das Ziel der Tragödie definierte, Gefühle eine zentrale Rolle. Dabei geht es nicht um die dargestellten Gefühle und auch nicht um die gefühlvolle Darstellungsweise, noch weniger um die Gefühle des Autors, denen Ausdruck verliehen werden kann, sondern um die Gefühle der Leser und Zuschauer, die geweckt werden sollen. Zwar wurde die Katharsis im Laufe der Jahrtausende unterschiedlich interpretiert, doch die Meinungen divergieren vor allem bezüglich der Art der Gefühle, die erweckt werden sollten, und bezüglich des Objekts der Reinigung, also bezüglich der Frage, was wovon gereinigt werden soll: der Zuschauer von den Gefühlen oder seine Gefühle selbst. Mitte des 19. Jahrhunderts begann jedoch ein Prozess der „Abkühlung“ in den Künsten. Die Gefühle, die noch in der Zeit der Romantik eine bedeutende Rolle spielten, wurden aus der Literatur langsam verdrängt, sowohl was die dargestellten Gefühle wie auch was die Darstellungsweise anging, und zwar zu Gunsten einer nüchternen Betrachtung der Lebenswelt. Die realistische Schreibweise und später der Naturalismus betonten, gestützt von den Theorien der Positivisten, die sozial-historischen, ökonomischen und biologischen Aspekte der Lebenswelten, die sie distanziert und mit einer fast wissenschaftlichen Akribie zu beschreiben suchten. Diese Abkehr von den Emotionen, die traditionell zu den Hauptthemen der Literatur gehörten, wurde als ein äußeres Zeichen von künstlerischer Modernität betrachtet, die der Modernisierung und Technisierung der Welt entsprechen sollte. Sie ging Hand in Hand mit Verschiebungen in den dominierenden Emotionsdiskursen der Zeit, die einerseits die Emotionen zum Untersuchungsobjekt von Naturwissenschaften machten, andererseits das Vokabular, das von den Geisteswissenschaften verwendet wurde, um Emotionen zu theoretisieren, einer weithin akzeptierten Vorstellung von Rationalität anpassten. Indem die Emotionen zum Gegenstand der Medizin unter anderen Wissenschaften wurden, wurde auch das Interesse wie auch die Vorstellung von Gefühlswelten verschoben, und zwar von Gefühlen als Manifestationen geistigen Lebens zu Emotionen als Folgeerscheinungen körperlicher Prozesse. Konkret heißt das zum Beispiel die Verschiebung des Interesses von der Liebe als Gefühl hin zur Sexualität, zu ihren Ursprüngen und Ausprägungen.

Diese Verschiebung ist auch im geisteswissenschaftlichen Diskurs zu beobachten, sie findet aber auch in der Literatur selbst statt, die immer offener Sexualität thematisiert, die Gefühle jedoch ausblendet, soweit sie sich nicht mit sich selbst und ihrer eigenen Form beschäftigt. Die Verschiebung im Bereich der Geisteswissenschaften markiert am deutlichsten Siegmund Freuds Psychoanalyse, die eine Brücke zwischen der medizinischen und der geisteswissenschaftlichen Erforschung von Sexualität schlägt. Freud, dessen Theorien bis heute nicht wissenschaftlich einzuordnen sind, führte gleichzeitig mit dem Untersuchungsobjekt Sexualität auch eine Sprache dafür ein, die sich an dem Vokabular der 
Wirtschaftswissenschaften orientiert. Neben der Medizin, von der er sich in seinen Theorien entfernte, galt die Ökonomie als Garant von Rationalität, gesundem Menschenverstand und distanzierter Emotionslosigkeit. Die Einführung des wirtschaftswissenschaftlichen Vokabulars bürgte dafür, dass die Sexualität im Rahmen dieser Theorien nüchtern und rational, also quasi wissenschaftlich untersucht wird. So spricht Freud über „Libidoökonomi““ (Freud 1991, 118), Jacques Lacans Theorie kreist um den Begritt des Mankos (manque), Jean-François Lyotard greift auf Freuds Terminologie zurück und schreibt über „Libidinöse Ökonomie" und Theoretikerinnen, die von Foucault und Deleuze ausgehend gegen Lacan argumentieren, fordern eine Theorie des Begehrens, die nicht mehr auf dem Konzept des Mankos und der Negativität, sondern auf Fülle und Reichtum beruhen würde, also eine Ökonomie des Überflusses, nicht des Mankos, wie Rosi Braidotti es formulierte (vgl. Braidotti 2000, 15).

Doch wenn auch Freud das Vokabular der Wirtschaft in die Diskussion der Sexualität einführte, hat die Verwendung von ökonomischen Denk- und Erklärungsmustern in Bezug auf Emotionen durchaus Tradition in der Geschichte des westlichen Denkens. Schon Platon erklärt im Symposion die Liebe mit einem Denkmodell, das auf dem wirtschaftlichen Begriff der Nachfrage basiert: Die Liebe erklärt er als Begehren von etwas, das man nicht hat. Schon bei Platon ist Eros, ähnlich wie bei Lacan, von einem inhärenten Mangel gezeichnet, das sein Begehren nach dem Schönen und Guten hervorbringt (vgl. Platon 199c-201d). Doch nicht nur im Rahmen der idealistischen Denktradition werden ökonomische Erklärungsmodelle für Emotionen bedient, auch die materialistisch orientierten Denker gebrauchen solche Modelle. So erklärt Karl Marx in seinen Frühen Schriften die Liebe als eine Art Tauschgeschäft, ein Geben und Nehmen (vgl. Marx 1971,301). Dieser vereinfachende, nüchterne Blick, den Marx auf die Liebe wirft, entzaubert die Liebe und macht sie zu einer Randerscheinung, die keiner weiteren Diskussion bedarf. Damit weist er seinen Anhängern, wie auch Bertolt Brecht, den Weg zu einer nüchternen, unsentimentalen Behandlung von Gefühlen, die nun nicht nur ein Ausdruck der Modernität ist, sondern auch ein politisches Vorzeichen bekommt. So wird der Emotionsdiskurs auch ideologisiert. Doch die Vermischung von Elementen verschiedener Denktraditionen sowie die Rationalisierung, Ökonomisierung und Ideologisierung der Emotionsdiskurse, werden Brecht Schwierigkeiten bereiten in seinem Versuch, gegen Aristoteles zu argumentieren.

Brecht bekämpfte das aristotelische Theater aus ideologischen und politischen Gründen. So fasste es schon Hans Mayer zusammen: „Brecht gedachte seine Dramenkunst und Bühnenkunst sehr deutlich gegen die Prinzipien der aristotelischen Poetik zu stellen, das ist bekannt. Er selbst wurde nicht müde, es immer wieder zu unterstreichen." (Mayer 1986, 33f) Den entscheidenden Unterschied 
zwischen Brecht und Aristoteles sieht Mayer in ihrer Konzeption der Funktion von Dramatik: „Die Funktion des Dramas begrenzt sich bei Aristoteles wie bei Lessing auf die Dauer des Theaterabends, die Geschlossenheit des Bühnenraums. Die Funktion der Brechtdramatik strebt über den Theaterabend und Zuschauerraum hinaus, will den Menschen belehren, auf Widersprüche des gesellschaftlichen Seins hinweisen, Erkenntnisprozesse auslösen, klares und einsichtsvolles Handeln im Leben provozieren." (Mayer 1986, 37)

Diese Argumentation von Mayer trifft in Bezug auf Brecht sicherlich zu, problematischer erscheint sie jedoch in Bezug auf Aristoteles, denn auch für ihn ist die Tragödie mit ihrem Bestreben, eine reinigende Wirkung auf die $\mathrm{Zu}$ schauer auszuüben, auf ein Ziel ausgerichtet, das außerhalb des Textes und des Theaters liegt. Dementsprechend ist es nicht verwunderlich, dass für Brecht der Angelpunkt seiner Kritik an der Poetik von Aristoteles gerade die Katharsis war. Im Gegensatz zu Mayers verkürzter Darstellung ist der Unterschied zwischen diesen zwei Vorstellungen der Dramenfunktion nicht so groß; deshalb bemühte sich Brecht in verschiedenen theoretischen Texten, diesen Unterschied zu präzisieren. Was das Brechtsche Motiv für den Kampf gegen Aristoteles angeht, beschreibt es Mayer recht überzeugend: „Brecht meinte von jeher die geschichtliche Gestalt des Aristoteles. Er lehnte nicht bloß die Poetik des Aristoteles ab [...] sondern vor allem seine Politik. Das konservative, auf Maß, Mitte, Ausgleich gerichtete System des Philosophielehrers Alexanders des Großen war ihm tief verdächtig." (Mayer 1986, 38)

Brecht hatte also politische und ideologische Gründe, die Bedeutung der Gefühle in der Dramentheorie und im Theater zu verringern und er hatte eben solche Gründe, sich von Aristoteles zu distanzieren. Hinzu kam, als drittes Kampffeld, Brechts Widerstand gegen die Praxis des bürgerlichen Theaters, die unter Berufung auf die Lessing'sche Poetik-Übersetzung auf die Erregung von Mitleid und Furcht ausgerichtet war. Diese drei Anliegen kommen zusammen, wenn Brecht gegen Aristoteles argumentiert, doch dabei argumentiert er, so die These, die im Folgenden weiter ausgeführt werden soll, vor allem gegen ein figurenzentriertes psychologisches Theater des späten Bürgertums.

Zur Katharsis kommt der Zuschauer, so Brecht, ,auf Grund eines eigentümlichen psychischen Aktes, der Einfühlung des Zuschauers in die handelnden Personen“ (Brecht 1993, 171). Die Katharsis, wie Brecht sie versteht, schließt kritische Rationalität aus: „Eine völlig freie, kritische, auf rein irdische Lösungen von Schwierigkeiten bedachte Haltung des Zuschauers ist keine Basis für eine Katharsis." (Brecht 1993, 172) Diese kritische Haltung aber ist wichtig im Klassenkampf in der Zeit des Hochkapitalismus. Brecht lässt in dem kurzen Text Kritik der „Poetik“ des Aristoteles (Brecht 1993, 171f) die Möglichkeit offen, dass der Verzicht auf Einfühlung nur zeitweilig sei, eben an den Hochkapitalismus 
gebunden, den es zu überwinden gilt. Doch nicht nur der Klassenkampf erforderte die Zurückstellung der Gefühle zu Gunsten einer kritischen und praktischen Vernunft. In der Zeit von 1933 bis zum Ende des Zweiten Weltkriegs, also in der Zeit der nationalsozialistischen Herrschaft, organisierten die Nationalsozialisten die so genannten Thing-Spiele, die darauf ausgerichtet waren, die Massen emotional zu mobilisieren, sie zu begeistern und zu manipulieren. Eine kühle, distanzierte Haltung des Publikums war so auch notwendig, um Brechts marxistisches Theater von den nationalsozialistischen Spektakeln zu differenzieren, so erhielt es neben dem ideologischen auch ein politisches Vorzeichen.

Das Abkühlen der Kunst, das für Brecht so wichtig war, begann, aus den bereits erwähnten Gründen, schon im 19. Jahrhundert. In der Literatur ist es besonders in der Prosa, vor allem im Roman, zu beobachten, wahrscheinlich wegen seiner relativen Flexibilität und der vermittelnden Instanz des Erzählers, die zwischen den dargestellten Figuren und dem Leser Distanz erschaffen kann. Doch für die Dramatik ist dieser Prozess schwieriger. Zwar lässt sich schon sowohl in dem Dargestellten als auch in den Darstellungsmitteln ein Zurücknehmen von Emotionalität auch im Drama beobachten, doch es bleibt nach wie vor wichtig, bei den Zuschauern eine emotionale Reaktion hervorzurufen, wenn auch durch Understatement. Die emotionale Reaktion des Publikums im Theater aufzugeben, ist aus zwei Gründen ein schwieriges Unterfangen. Das erste Problem betrifft die Theorie. Am Beginn des 20. Jahrhunderts gibt es keine Dramentheorie, die auf das Erregen der Gefühle des Publikums verzichtete. Eine neue Theorie müsste formuliert werden, die gegen die von Aristoteles bestimmte Tradition das Ziel der Dramatik neu definieren würde. Das zweite Problem dabei ist praktisch und lässt sich leicht beschreiben, jedoch schwer lösen: Werden die Emotionen des Publikums nicht angesprochen, so gehen das Interesse und die Aufmerksamkeit verloren. Geht das Publikum verloren, so gibt es kein Theater.

Brecht versuchte beide Probleme zu lösen. In der Theorie konzentrierte er sich auf die Dramenrezeption und entwickelte das Konzept des epischen Theaters, wo das Gefühl, das er dem aristotelischen Theater zuschrieb, zurücktrat zu Gunsten der Ratio. Dies wird aus der Tabelle ersichtlich, die er in dem Text Das moderne Theater ist das epische Theater: Anmerkungen zur Oper Aufstieg und Fall der Stadt Mahagonny veröffentlichte (Brecht 1957). Um das zu erreichen, wandte er im Drama einige der Textstrategien an, die sich bereits in der Epik zur Abkühlung der Emotionen bewehrt hatten. Der erste wesentliche Aspekt war die epische Struktur, die er einführte, der zweite die epische Spielweise, bei der der Schauspieler zeigt, wie er sich die Figur "denkt“ (Brecht 1957, 154). Der Schauspieler distanziert sich von der Rolle. Er spielt und gleichzeitig zeigt er, dass er spielt. Er funktioniert als vermittelnde Instanz, ähnlich dem Erzähler in der Prosa. Andere Mittel der Verfremdung, die Brecht verwendete, waren die Songs, die den 
Zusammenhang der Geschichte unterbrachen. Die Verwendung von Musik, die zwar die Identifikation des Zuschauers erschweren soll, hilft jedoch auch, seine Aufmerksamkeit zu fesseln. So weit, so gut.

Brecht aber war ein Theaterregisseur und wusste, dass das Theater nicht nur die Ratio ansprechen und auf seine didaktische Funktion reduziert werden darf. Schon in den Anmerkungen zur Oper Aufstieg und Fall der Stadt Mahagonny fügt er eine Fußnote hinzu, in der er betont, dass die im Schema aufgelisteten Gegenüberstellungen nicht als „absolute Gegensätze“ zu verstehen sind, sondern dass „innerhalb eines Mitteilungsvorgangs das gefühlsmäßig Suggestive oder das rein rationell Überredende bevorzugt werden“"kann (Brecht 1957, 19). Später versuchte er in anderen Texten zum Theater immer wieder den Eindruck zu korrigieren, der durch die graphische Gegenüberstellung in dem erwähnten Schema erweckt wurde und die Austreibung der Emotion aus seinem Theater implizierte. In dem Text Vergnügungstheater oder Lehrtheater? behauptet er sogar, dass, wer Vernunft und Gefühl als Gegensätze sieht, weder große Gefühle noch wirkliche Vernunft kenne. Sowohl Vernunft als auch Gefühl seien im Zeitalter des Kapitalismus „entartet“. „Die aufsteigende neue Klasse hingegen und jene, die mit ihr zusammen kämpfen, haben es mit Vernunft und Gefühl in großem produktivem Widerspruch zu tun." (Brecht 1957, 73)

Gleichzeitig mit dieser versuchten Rehabilitation von Gefühlen begann Brecht aber auch, in seinem theoretischen Gebilde die Sinnlichkeit und die Lust zu betonen. Diese ist jedoch nicht Selbstzweck, sondern dient dem Lernen. Es geht also um „lustvolles Lernen, fröhliches und kämpferisches Lernen“, um „amüsantes Lernen“ (Brecht 1957, 66). Das Theater muss Spaß machen und wenn es moralische Lehren vermitteln will, muss es sie vergnüglich machen, „und zwar den Sinnen vergnüglich“ (Brecht 1957, 131). Wo es um Lust, Spaß oder Freude in Verbindung mit dem Lernen geht, findet Brecht auch die aristotelische Poetik akzeptabel: „Solange der Aristoteles (im vierten Kapitel der Poetik) ganz allgemein über die Freude an der nachahmenden Darstellung spricht und als Grund dafür das Lernen nennt, gehen wir mit ihm." (Brecht 1993, 172) Genauer betrachtet, geht Aristoteles a priori davon aus, dass Lernen Freude macht. Es muss gar nicht auf besondere Weise vermittelt werden, denn es ist an sich schon ein Vergnügen: „Das Lernen bereitet nicht nur den Philosophen größtes Vergnügen, sondern in ähnlicher Weise auch den übrigen Menschen (diese haben freilich nur wenig Anteil daran)“(Aristoteles 1448b). Ein vergnügliches Lernen wäre demnach eigentlich schon eine Tautologie.

In seinem Essay Kleines Organon für das Theater betont Brecht auch die zentrale Bedeutung der „Fabel“, die er als „Herzstück der theatralischen Veranstaltung“ bezeichnet und als „Gesamtkomposition aller gestischen Vorgänge“ definiert. Sie zeigt das, „was zwischen den Menschen vorgeht“, woraus der Zuschauer alles 
ablesen kann, „was diskutierbar, kritisierbar, änderbar sein kann“ und dem Publikum Vergnügen bereitet (Brecht 1957, 165). Während er für sein Theater also die vorrangige Bedeutung der Fabel hervorhebt, sieht er in der Poetik von Aristoteles vor allem die Bedeutung der Figuren hervorgehoben, wie er in anderen Texten ausführt. Die Katharsis, das Ziel der Tragödie, werde, so Brechts Interpretation der Poetik, durch die „Einfühlung des Zuschauers in die handelnden Personen“ (Brecht 1993, 171) erreicht. So heißt es in dem Aufsatz Über die Verwendung von Musik für ein episches Theater auch: „In der aristotelischen Dramatik wird der Held durch die Handlungen in Lagen versetzt, in denen er sein innerstes Wesen offenbart. Alle gezeigten Ereignisse verfolgen den Zweck, den Helden in seelische Konflikte zu treiben." (Brecht 1957, 243) Emotionen und Vergnügen schöpft der Zuschauer in der Theorie des epischen Theaters von Brecht aus der Fabel, der „Gesamtkomposition aller gestischen Vorgänge“, während sie bei Aristoteles, wie Brecht dessen Poetik interpretiert, durch die Einfühlung mit den Figuren erreicht werden sollten. Diese Auslegung der Poetik erscheint, wenn man den Text genau betrachtet und dabei auch die neueste Forschung berücksichtigt, etwas eigenwillig.

Aristoteles bezeichnet die „Zusammensetzung der Geschehnisse“, die „Nachahmung von Handlung“ als Mythos. Dies ist der „wichtigste Teil“ der Tragödie, die ohne Handlung nicht zustande käme, „wohl aber ohne Charaktere“. (1450a) Ein weiteres der sechs Elemente, aus denen die Tragödie besteht, ist die Erkenntnisfähigkeit (1450a). Schon hier wird deutlich, dass auch für Aristoteles das Theatererlebnis nicht nur auf Gefühlen und sinnlichen Wahrnehmungen basiert. Doch der Mythos, die Handlung, bleibt für ihn das wichtigste Element der Tragödie und somit ist er für ihn auch der wichtigste Auslöser von Emotionen: „Nun kann das Schauderhafte und Jammervolle durch die Inszenierung, es kann aber auch durch die Zusammenfügung der Geschehnisse selbst bedingt sein, was das Bessere ist und den besseren Dichter zeigt. Denn die Handlung muß so zusammengefügt sein, daß jemand, der nur hört und nicht auch sieht, wie die Geschehnisse sich vollziehen, bei den Vorfällen Schaudern und Jammer empfindet.“ (1453b) Von Einfühlung in die Charaktere ist hier nicht die Rede. Wohl aber präzisiert Aristoteles, wie die Geschehnisse zusammengesetzt werden sollten und noch genauer führt er drei Beispiele auf, wie sie nicht zusammengesetzt werden sollten, damit sie eine emotionale Reaktion beim Publikum hervorrufen, die über das Empfinden von Abscheu hinausginge. Was nicht gezeigt werden sollte ist der Fall eines makellosen Mannes, der Aufstieg eines Schufts und der Fall eines ganz schlechten Mannes. Das erste schockiert nur, das zweite ist unakzeptabel, das dritte selbstverständlich (vgl. 1452b-1453a). Für die Katharsis ist das Hervorrufen von Emotionen nur der erste Schritt, der zweite ist die Reinigung. Für diese aber ist offensichtlich wichtig, dass die Zuschauer verstehen, wie und warum 
etwas passiert, also dass sie die kausalen Zusammenhänge und die ethische Dimension der dramatischen Handlung verstehen. Auch die neuesten Forschungen weisen darauf hin, dass sich für Aristoteles das Erregen von Emotionen und eine kritische, rationale Haltung nicht ausschließen.

So bezeichnet Adelheid Stephan die Katharsis bei Aristoteles als „rezeptionsästhetisches emotives und erkenntnistheoretisches Prinzip der Tragödie“ (Stephan 2012, 173). Doch genauer geht auf die Problematik der Emotionserregung bei Aristoteles Christof Rapp ein. Er macht darauf aufmerksam, dass Aristoteles in seiner Rhetorik zwischen kunstfremder und kunstgemäßer Emotionserregung unterscheidet. Von der kunstfremden sage Aristoteles, „sie verbindere ein wirkliches Urteil, während er von der kunstgemäßen Erregung von Emotionen sagt, sie modifiziere das Urteil der betroffenen Zuhörer“. (Rapp 2007,158). Weiter stellt er die These auf: „Möglicherweise meint er daher, daß seine Methode der Emotionserregung über die Modifikation der Urteile zur Überzeugungsbildung beiträgt, während die konventionelle Bemühung, den Hörer durch Emotionen von der Sache abzulenken, eher dahin geht, eine Überzeugungsbildung zu verhindern“ (Rapp 2007, 158). Vor allem wichtig ist jedoch seine Feststellung, für Aristoteles gehöre „die Emotionserregung, wie er sie selbst konzipiert, zum Bereich des Gedankens oder der Gedankenführung (diánoia) und stellt daher eine inhaltsbezogene Weise der Überzeugung dar." (Rapp 2007, 158) So sei, so Rapp, auch die Emotionserregung durch die Handlungszusammenhänge in der Tragödie dem Bereich des Gedankens zuzurechnen, der zur Urteilsbildung führt (vgl. Rapp 2007, 162). Wichtig dabei aber ist doch, der Redner muss „durch das, was er sagt, den Zuhörer glauben machen, daß etwas der Fall ist“ (Rapp 2007, 159). Es geht also auch um die Art und Weise, wie etwas dargestellt wird, damit es glaubwürdig ist.

Ich will nicht ausschließen, daß in der Tragödie auch Rhythmus, Melodik, Aufführung, Inszenierung eine emotionsrelevante Wirkung entfalten; erstens jedoch wertet Aristoteles diese gegenüber der Handlung in der Tragödientheorie genauso ab, wie er in der Rhetorik den mündlichen Vortrag und die sprachliche Form gegenüber der diánoia abwertet; und zweitens erscheint die Handlung beim Erzielen der tragödienspezifischen Wirkung autonom. (Rapp 2007, 162)

Für Aristoteles ist also die Handlung oder der Mythos das wichtigste im Drama, wie für Brecht die Fabel. Die Ratio, die Brecht der aristotelischen Emotion gegenüberstellt, ist auch bei Aristoteles ein bedeutender Teil der Tragödie und die kunstgemäß erregten Gefühle schließen eine kritische Urteilsbildung nicht aus. Es scheint, dass sich Brecht immer mehr an die aristotelische Poetik annäherte, je 
mehr er sich von ihr loszulösen versuchte. Noch offensichtlicher als in der Theorie passierte dies in seinem literarischen Schaffen, was von der Literaturwissenschaft bereits beobachtet und behandelt wurde. Gerade in der Zeit zwischen 1937 und 1939, als er an seiner Kritik der „Poetik" arbeitete, entstanden auch zwei Theaterstücke, die als „aristotelisch“ gelten, und zwar Die Gewebre der Frau Carrar und Leben des Galilei. Hans Mayer, der Brecht verteidigt, räumt ein, dass es in diesen Stücken zwar keine Katharsis gäbe, aber „eine spezifische Schwierigkeit dialektischer Theaterarbeit" (Mayer 1986, 42) dabei sichtbar werde. Die Verteidigung stützt er vor allem durch die Hervorhebung von Brechts Verständnis der Notwendigkeit einer „vielfältigen, formenreichen, stets aber dialektischen Bühnenpraxis.“ (Mayer 1986, 42)

Obwohl sich Brecht weder in seiner Theorie noch in seiner literarischen Praxis von Aristoteles vollkommen lösen konnte, suchte er nach einer Möglichkeit, sich von ihm zu distanzieren. Die Formulierungen in dem kurzen Text zur Kritik an der Poetik klingen schon fast krampfhaft verzweifelt, denn auch die Einfühlung, die er Aristoteles und den Theaterbesuchern der griechischen Antike zuschreiben möchte, kann er nicht nachweisen:

Einfühlung beim Aristoteles. Nicht daß wir beim Aristoteles als Art der Entgegennahme des Kunstwerks durch den Zuschauer die Einfühlung finden, die heute als Einfühlung in das Individuum des Hochkapitalismus vorkommt. Dennoch haben wir bei den Griechen, was immer wir uns unter der Katharsis, die unter uns so fremden Umständen vor sich ging, vorstellen mögen, als deren Basis irgendeine Art von Einfühlung zu vermuten. (Brecht 1993, 172)

In dem Abschnitt, indem Brecht „irgendeine Art“ von Einfühlung bei den Griechen vermuten will, obwohl er der Antike kein neuzeitliches Individuum oder Subjektkonzept unterstellen kann, wird deutlich, dass er vor allem an der „Einfühlung in das Individuum des Hochkapitalismus“ Anstoß nimmt. Es wird deutlich, dass er die neuzeitliche, bürgerliche Interpretation der aristotelischen Poetik bekämpft, die ihr eigenes Interesse an Individualität, an intrasubjektiven Vorgängen und moderner Psychologie auf die antike Poetik projizierte. Er versucht in anderen Texten die Aufmerksamkeit des Theaters wieder auf die intersubjektiven Vorgänge zu lenken, auf das zwischenmenschliche Geschehen, also auf das, was Peter Szondi in seiner Mitte der 1950er Jahre veröffentlichten Theorie des Theaters als „die Dichtungsform des je gegenwärtigen zwischenmenschlichen Geschehens" definiert (Szondi 1963, 74). Brecht hat so ungewollt jene Dimension der Poetik herausgearbeitet, die im deutschsprachigen bürgerlichen Theaterdiskurs ab Mitte des 19. und zu Beginn des 20. Jahrhunderts übersehen oder eigenwillig interpretiert worden sind. In dieser Zeit dominierte das Interesse 
an dem „psychologischen Aspekt des Katharsisproblems“ und „die emotive Deutung der Katharsis“ (Luserke 1991, XIII), die zwar schon seit Jakobus Pontanus angelegt war, aber mit Jacob Bernays Studien große Resonanz erfuhr. So werden in dieser Zeit vor Brecht in Aufsätzen zur Katharsis vermehrt Begriffe verwendet wie das „psychologische Faktum“ (Weil 1991, 78), „Entladung der Affekte“ (Volkelt 1991, 157) oder es werden Vergleiche zu Breuers und Freuds Behandlung der Hysterie angestellt (vgl. Berger 1991, 139).

Brechts Betonung der Ratio und der Abkühlung von Gefühlen war also gegen die Emotionen erhitzenden nationalsozialistischen Spektakel gerichtet, die zwar mit dem aristotelischen Theater nichts gemeinsam haben (sie bezogen sich eher auf ein pervertiertes Verständnis germanischer Mythologie) und in seinem Sinne eher einem kunstfremden Erregen von Emotionen zur Verhinderung der Urteilsbildung entsprechen. Sie kommen ohne Brechts Ratio und ohne aristotelische diánoia aus. Brechts Kritik an der Einfühlung hingegen richtet sich gegen das psychologisierende, plüschgepolsterte, warme bürgerliche Theater, das sich auf intrasubjektive Vorgänge konzentrierte und das Zwischenmenschliche, Soziale und Politische ignorierte, wodurch es in Brechts Sicht konservativ erschien, da es der privilegierten Klasse vorbehalten belieb, die sich mit sich selbst beschäftigte, um sich nicht mit realen gesellschaftlichen Problemen auseinandersetzen zu müssen. Die Brecht-Forschung hat diese Ansätze zwar thematisiert, doch hat sie sie einerseits zu wenig differenziert, wie es Karl-Heinz Ludwig hervorhebt, der meint, dass Brecht die Einfühlung zur Theatralik des Faschismus zähle (vgl. Ludwig 1975, 156), andererseits aber vor allem in die philosophische Tradition einzuordnen versucht, wie Franz Norbert Mennemeier und Michael Thiele. Mennemeier bemerkt, dass Brecht eine in Psychologie versandende unpolitisch-illusionistische Kunst kritisiert, wodurch er sich wieder Platons Kunstkritik annähert (Mennemeier 1979, 145f). Thiele hingegen sieht darin keine Rückkehr zu Platon, sondern eher eine Kritik an einer auf Einfühlung beruhenden Kunstauffassung, die Brecht zwar als „aristotelisch“ bezeichnet, die sich aber eher an Hegels Konzeption der romantischen Kunstform orientiert (Thiele 1991, 148).

So formierte sich Mitte des 20. Jahrhunderts vor dem Hintergrund historischer Ereignisse eine auf die Kunst bezogene Temperaturskala, in der Wärme der konservativen, bürgerlichen Kunst und Kälte der modernen, progressiven, meistens politisch linksorientierten Kunst zugeschrieben wurde, während populistische (potentiell rechtsorientierte) Spektakel als heiß galten. Diese Temperaturzuschreibungen samt den politischen Vorzeichen sind so suggestiv, dass sie bis in die Gegenwart nachwirken. So ordnet auch Hans-Thies Lehmann in seiner Theorie des postdramatischen Theaters den negativ konnotierten Begriff Wärme einem veralteten, an psychologischer Darstellung menschlicher Schicksale orientierten Theater zu, während Kälte das moderne beziehungsweise postmoderne Theater auszeichnet. 
Der Kälte, die im Formalismus des postdramatischen Theaters verortet wird, wird hier nicht explizit ein politisches Vorzeichen zugeschrieben, doch durch dessen Modernität kommt auch ihr eine kunstimmanent positive Wertung zuteil:

Durch die Beteiligung lebender Menschen wie auch durch die jahrhundertealte Fixierung auf bewegende Menschenschicksale eignete dem Theater eine gewisse 'Wärme'. Dem haben zwar klassische Avantgarden, das epische und dokumentarische Theater schon weitgehend den Garaus gemacht. Dennoch ist der Formalismus des postdramatischen Theaters ein qualitativ neuer Schritt und löst noch immer Ratlosigkeit aus. Er kann für den, der auf die Darstellung menschlicher im Sinne von psychologischer Erfahrungswelten wartet, eine schwer erträgliche Kälte manifestieren. (Lehmann 1999, 161f)

Was die Ablehnung der psychologischen Darstellung von Figuren angeht, ist das postdramatische Theater von Lehmann nicht besonders innovativ, da diese bereits von Brecht abgelehnt, von Aristoteles hingegen nie gefordert wurde. Diese Ablehnung richtet sich noch immer gegen ein bürgerliches Theater, das auf einer dem 19. Jahrhundert geschuldeten eigenwilligen Interpretation der Poetik von Aristoteles, insbesondere des Begriffs der Katharsis, beruht. Die Differenz, die das postdramatische Theater einführt, liegt vielmehr in seinem Umgang mit der Handlung. Im Vergleich mit dem postdramatischen Theater ist Brechts Theater dramatisch gerade in seiner Betonung der Handlung. Auch Birgit Haas bezeichnet es als dramatisch, während sie in der Postmoderne eine Radikalisierung von Brechts Theaterästhetik sieht, die dadurch zerstört wird. Für sie ist jedoch das wichtigste Kennzeichen des dramatischen Theaters „das Streben nach einem ganzheitlichen Kunstwerk, welches die Erfahrungen eines dialektischen Bezugs von Kunst und Realität einbezieht" (Haas 2007, 47).

Abschließend kann festgestellt werden, erstens, dass sich Brecht, je mehr er ausdrücklich gegen Aristoteles argumentiert, umso stärker einer genauen Lesart seiner Poetik annähert, und zweitens, dass sich das sogenannte postdramatische Theater, das sich ausdrücklich von Aristoteles und Brecht distanziert, sich als kalt bezeichnet, obwohl sich die Kälte als Charakteristikum eines modernen, progressiven, anti-bürgerlichen und anti-faschistischen Theaters gerade durch Brechts Annäherung an Aristoteles unter besonderen politischen und ideologischen Verhältnissen Mitte des 20. Jahrhunderts entwickelte. Doch es ist nicht das Ziel des vorliegenden Artikels, die Besonderheiten des sogenannten postdramatischen Theaters zu bestimmen, sondern nur auf bestimmte Widersprüche in der Dramentheorie des 20. Jahrhunderts hinzuweisen, die durch eine überkommene implizite Wertung bestimmter dramaturgischer Techniken, in der jedes Erregen emotioneller Reaktionen des $\mathrm{Pu}$ blikums ein kunstimmanent und politisch abwertendes Vorzeichen bekommt, die Möglichkeiten für die Entwicklung neuer Schreibweisen begrenzt. 


\section{Literaturverzeichnis}

Aristoteles: Poetik. Stuttgart: Reclam 1982.

Berger, Alfred Freiherr von: „Wahrheit und Irrtum in der Katharsistheorie des Aristoteles". Luserke, Matthias: Die Aristotelische Katharsis: Dokumente ibrer Deutung im 19. Und 20. Jabrbundert. Hildesheim, Zürich, New York: Georg Olms Verlag 1991, S. 128-156.

Braidotti, Rosi: Feminizem/mi za začetnice/ke. Ljubljana: *f. 2000.

Brecht, Bertolt: Über experimentelles Theater. Frankfurt am Main: Suhrkamp 1970.

Brecht, Bertolt: Schriften zum Theater. Frankfurt am Main: Suhrkamp 1957.

Brecht, Bertolt: Werke. Berlin: Aufbau, Frankfurt am Main: Suhrkamp 1993. Bd. 22. Freud, Sigmund: Drei Abhandlungen zur Sexualtheorie. Frankfurt am Main: Fischer 1991.

Haas, Birgit: Plädoyer für ein dramatisches Drama. Wien: Passagen 2007.

Lehmann, Hans: Postdramatisches Theater. Frankfurt am Main: Verlag der Autoren 1999.

Ludwig, Karl-Heinz: Bertolt Brecht: Philosophische Grundlagen und Implikationen seiner Dramaturgie. Bonn: Bouvier 1975.

Luserke, Matthias: Die Aristotelische Katharsis: Dokumente ihrer Deutung im 19. Und 20. Jahrbundert. Hildesheim, Zürich, New York: Georg Olms Verlag 1991. Lyotard, Jean-Françoise: Libidinöse Ökonomie. Zürich, Berlin: diaphanes 2007.

Marx, Karl: Die Frühschriften. Stuttgart: Alfred Körner Verlag 1971.

Mayer, Hans: „Anti-Aristoteles“. Werner Hecht (Hg.): Brechts Theorie des Theaters. Frankfurt am Main: Suhrkamp 1986, S. 32-42.

Mennemeier, Franz Norbert: Brecht - Mutter Courage und ihre Kinder. Theo Buck (Hg.): Zu Bertolt Brecht. Parabel und episches Theater. Stuttgart: Klett-Cotta 1979.

Platon: Sämtliche Werke. Reinbek bei Hamburg: Rowohlt 2013.

Rapp, Christof: „Katharsis der Emotionen“. Martin Vöhler, Bernd Seidensticker (Hgg.): Katharsiskonzeptionen vor Aristoteles. Berlin: De Gruyter 2007 S. $149-172$.

Stephan, Adelheid: Die aristotelische Katharsis in rezeptionsästhetischer und erkenntnistheoretischer Perspektive. Hamburg: Verlag Dr. Kovač 2012.

Szondi, Peter: Theorie des modernen Dramas. Frankfurt/M. 1963.

Thiele, Michael: Negierte Katharsis: Platon - Aristoteles - Brecht. Frankfurt am Main (etc.): Peter Lang 1991.

Volkelt, Johannes: „Die Tragische Entladung der Affekte“. Luserke, Matthias: Die Aristotelische Katharsis: Dokumente ibrer Deutung im 19. Und 20. Jahrbundert. Hildesheim, Zürich, New York: Georg Olms Verlag 1991, S. 157-172. 
Weil, Henri: „Ueber die Wirkung der Tragödie nach Aristoteles“. Luserke, Matthias: Die Aristotelische Katharsis: Dokumente ihrer Deutung im 19. Und 20. Jahrbundert. Hildesheim, Zürich, New York: Georg Olms Verlag 1991, S. 69-79.

\section{Universität Ljubljana, Slowenien spela.virant@guest.arnes.si}

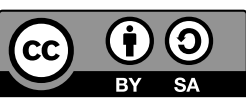

\section{O vlogi čustev v Brechtovi teoriji drame}

Članek na kratko oriše proces postopnega čustvenega »ohlajanja« literature, ki se je začel že sredi 19. stoletja in povzročil poseben problem v teoriji drame, ki je vse od Aristotelove Poetike temeljila na vzbujanju in očiščenju čustev. Brecht je v svoji teoriji epskega gledališča, kjer argumentira proti Aristotelu, poudaril vlogo razuma, ki naj bi nadomestil čustvovanje. Natančna analiza Brechtovih besedil in sodobne raziskave Aristotelove Poetike pa pokažejo, da se je Brecht upiral predvsem proti interpretacijam Poetike, ki so nastale v 19. stoletju in spremljale razvoj meščanske psihologizacije gledališča. S svojimi argumenti proti tako razumljeni Poetiki pa se je pravzaprav močno približal sodobnim branjem Aristotelove Poetike.

Ključne besede: Bertolt Brecht, Aristoteles, Poetika, teorija drame, teorija gledališča

\section{About the Role of Emotions in Brecht's Theory of Drama}

The article briefly decribes the process of gradual "cooling down « of literature, which began already in the middle of the 19th century and caused a special problem in the theory of drama that has been since Aristotle's Poetics based on the evocation and purgation of emotions.

Key words: Bertolt Brecht, Aristotle, Poetics, theory of drama, theory of theatre 